\title{
Charakterisierung von Filtermedien durch den Einsatz von Photozentrifugalfiltration (PZF)
}

\author{
Sebastian Boldt ${ }^{1}$, Philipp Lösch², Daniel Krause' ${ }^{1}$, Christian Weibel2, Dietmar Lerche1, Sergiy Antonyuk ${ }^{2}$ \\ ${ }^{1}$ LUM GmbH, Berlin \\ 2Lehrstuhl für Mechanische Verfahrenstechnik, Technische Universität Kaiserslautern \\ Kontakt:info@lum-gmbh.de
}

\section{Einleitung}

Für die Herstellung oder Weiterverarbeitung von Wertstoffen werden Dispersionen in vielen Industriezweigen filtriert. Die hierzu notwendigen Anlagen werden meist auf Basis aufwendiger und zeitintensiver Versuche im Labor- und/oder Technikumsmaßstab unter Berücksichtigung von Sicherheitsfaktoren ausgelegt. Für die Versuche werden häufig klassische Filternutschen in Form einer Druck- oder Vakuumfiltration eingesetzt [1 - 3]. Im Zuge der Entwicklung von Bio- und Nanotechnologie ergeben sich neue Herausforderungen, da in diesen Bereichen oft nur kleine und meist auch sehr teure Probenmengen zur Verfügung stehen. Weiterhin ist eine sorgfältige Auswahl des Filtermittels (FM) notwendig, da neben den hohen Anforderungen an die Stofftrennung auch die Wechselwirkungen der Inhaltsstoffe der zu filtrierenden Flüssigkeiten mit dem FM berücksichtigt werden müssen. Letztere beeinflussen auch die Haftung von Partikeln sowie die Adsorption von Inhaltsstoffen am FM, wodurch auch die Durchlässigkeit des FM und der Reinigungsaufwand beeinflusst werden. Insbesondere beim Einsatz von Membranen zur Filtration von Dispersionen (Suspensionen, Emulsionen sowie kolloidalen Lösungen) sind aufwendige Untersuchungsserien mit mehreren unterschiedlichen FM notwendig und üblich.

Ein Filtrationsexperiment unter Verwendung standardisierter Filternutschen (vgl. [3]) erfordert eine große Menge an Versuchssuspension $(300 \mathrm{ml}$ pro Versuch) und große Flächen an FM (45 mm bis 50 $\mathrm{mm}$ im Durchmesser). Der Einsatz solcher Probenmengen in der Bio- oder Nanotechnologie ist neben immensen Kosten auch mit hohem ökologischem Risiko verbunden, wenn Gefahrstoffe enthalten sind. Für die Entwicklung von FM sind kleine Filtermedienabmessungen ebenfalls von Vorteil.

Dieser Artikel berichtet über die Weiterentwicklung der analytischen Photozentrifugalfiltration (PZF), welches vorteilhaft eine geringe Menge an Filtrationsproben bzw. kleine Membrangrößen benötigt, um schnell eine Abschätzung/Optimierung für eine effektive Kombination Suspension-/Emulsion und Filtermedium in Anlehnung an konventionellen Methoden zu ermöglichen. Es können bis zu 12 Proben parallel in einem Zentrifugenlauf untersucht werden.

\section{Methoden und Materialien}

Als Basis der PZF dient eine analytische Photozentrifuge (LUMiSizer®, LUM GmbH).
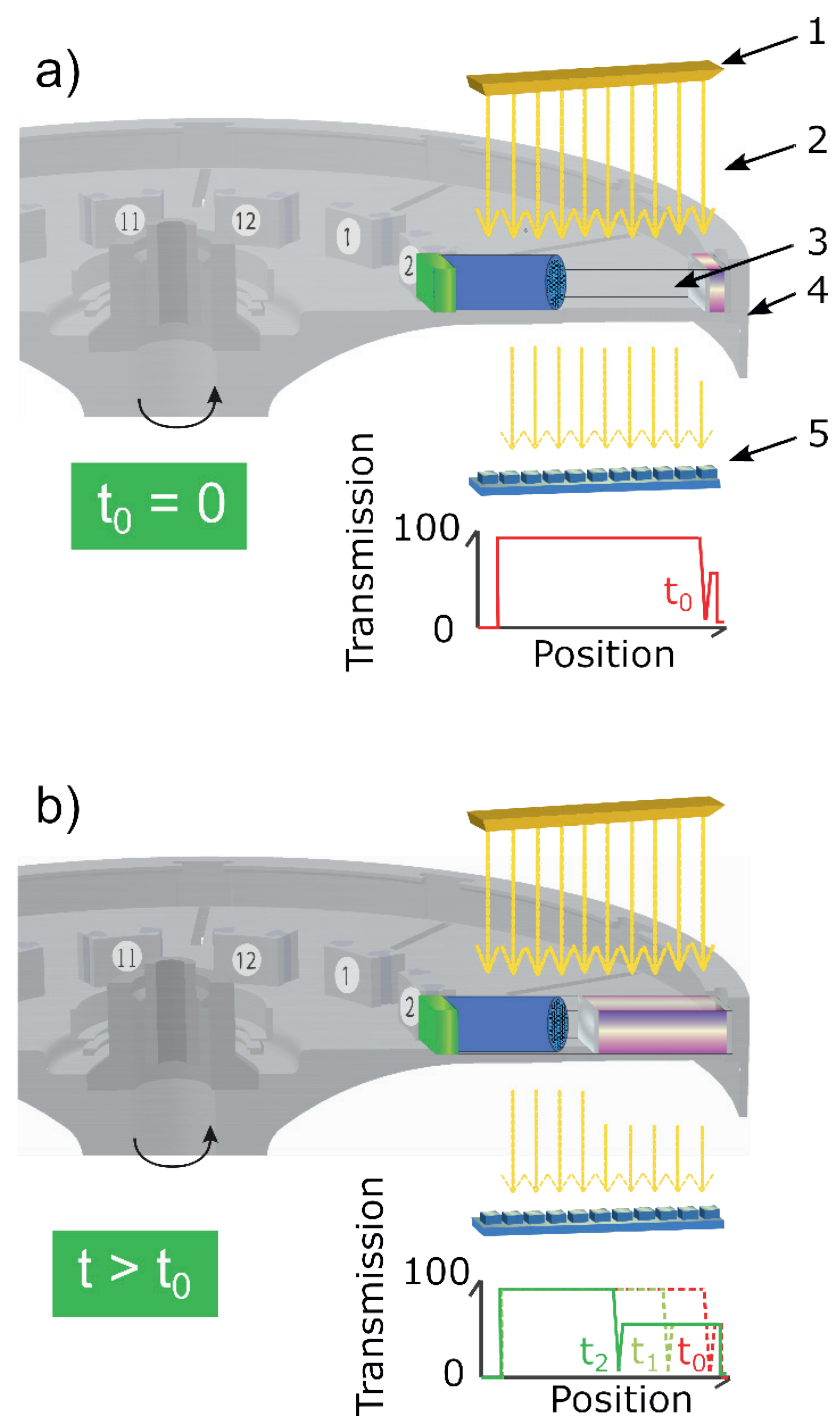

Abb. 1: Prinzip der orts- und zeitaufgelösten Extinktionsprofile in der analytischen Photozentrifuge (LUMiSizer®, LUM GmbH, Deutschland).

a) Transmissionsprofile zum Startzeitpunkt der Zentrifugation mit kleinem vorgelegten Filtratvolumen (rote Linie). b) Transmissionsprofile zu späteren Zentrifugationszeitpunkten (ocker-farbene und grüne Linie). (1) Lichtquelle; (2) paralleles NIR oder VIS Lichtbündel; (3) Filtrationsmesszelle; (4) Mehrprobenrotor; (5) Zeilendetektor 
Mittels der STEP-Technologie $®$ können in-situ ortsund zeitaufgelöste Extinktionsprofile automatisch aufgenommen werden. Abbildung 1 zeigt das Schema einer kommerziellen analytischen Photozentrifuge mit Mehrprobenrotor. Rotorgeschwindigkeiten bis zu 4000 Umdrehungen pro Minute und Temperierungen von 4 bis $60^{\circ} \mathrm{C}$ sind möglich. Die analytische Photozentrifugation ist weit verbreitet für die Dispersionscharakterisierung [4].

Linear parallelisiertes Licht durchleuchtet das Filtratreservoir der Filtrationsmesszelle, welche horizontal auf den Mehrprobenrotor platziert wird. An der Grenzfläche Luft-Filtrat bildet sich ein Meniskus aus, dessen Geometrie im Wesentlichen von der Oberflächenspannung des Filtrates (und dem Küvettenmaterial) abhängt. Die optische Abbildung des Meniskus ist ein starker, lokaler Transmissionsabfall. Abhängig der Filtratmenge ändert dieses Minimum seine Position mit fortschreitender Filtrationszeit. Der Sensor detektiert die gesamte Höhe des Filtratreservoirs mit einer örtlichen Auflösung von etwa 14 Mikrometern. Die Zeitintervalle können vom Anwender vor dem Versuchsstart festgelegt werden. Dadurch wird eine orts- und zeitaufgelöste Bestimmung des Filtratfüllstandes ermöglicht. Mithilfe der Detektion des Meniskus mittels STEP-Technologie, auch unter Berücksichtigung der Oberflächenspannung und der verwendeten Rotordrehgeschwindigkeit kann das Filtrationsverhalten automatisiert über die Zeit in-situ bestimmt werden.

In der PZF kommen speziell entwickelten Filtrationsmesszellen zur Untersuchung des Filtrationsverhaltens von Dispersionen und FM zum Einsatz (Abb. 2). Diese sind aus Edelstahl oder PEEK (Polyetheretherketon) gefertigt und bestehen aus einem zweiteiligen

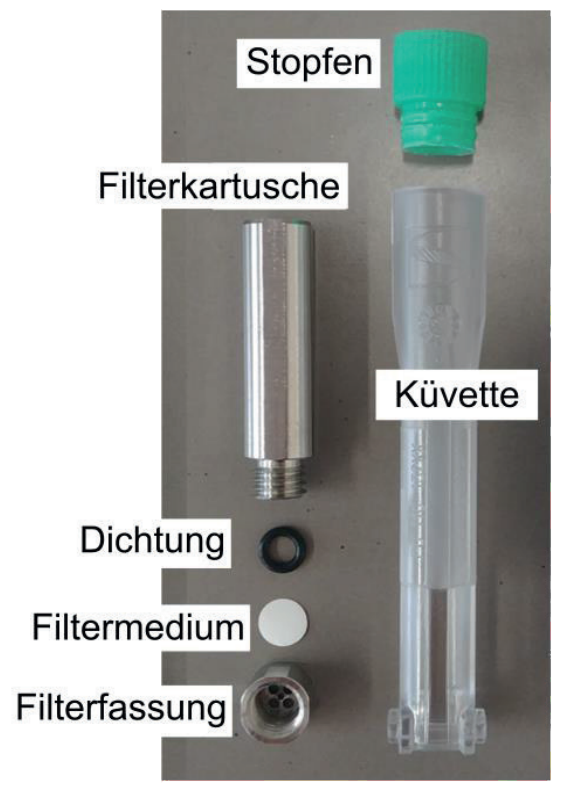

Abb. 3: PZF Messzelle bestehend aus einem zweiteiligen Filtrationsmodul und modifizierter Einwegküvette [5]

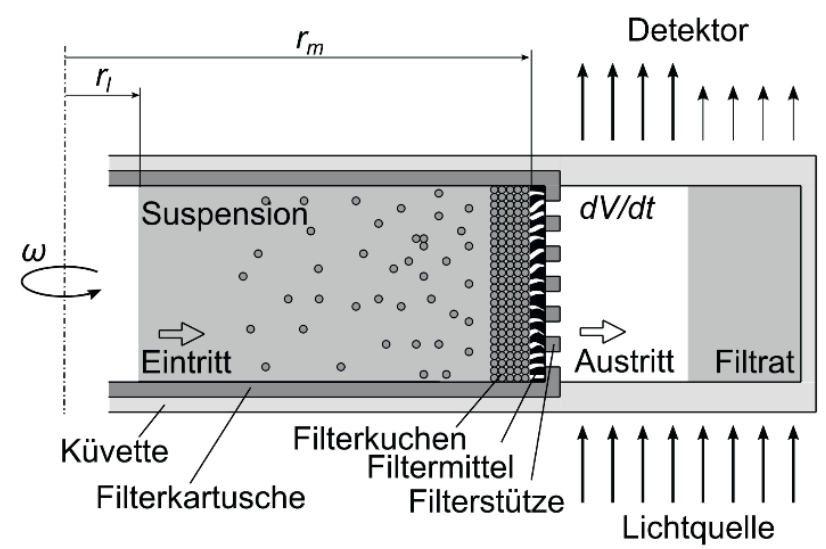

Abb. 2: Schema der Filterküvette im Zentrifugalfeld

Aufbau aus Kartusche und Filtermittelfassung. Das FM bzw. die Membran wird in der Fassung positioniert. Es können flache, kreisrunde FM von 7 Millimetern verwendet werden. Der Aufbau wird dann verschraubt und mittels eines Kautschukringes abgedichtet. Dieses Filtrationsmodul wird in einer modifizierte Einweg-Standardküvette (LUM GmbH, Deutschland) fixiert und mit einem Stopfen verschlossen. In dem Filtrationsmodul können Probenvolumina bis zu $1 \mathrm{ml}$ gefüllt werden.

Bei der Filtration im Zentrifugalfeld wird der Druck maßgeblich durch die Fluidsäule oberhalb des Filtermittels und der Zentrifugalbeschleunigung gesteuert. Mit fortschreitender Filtration (Abnahme des Suspensionsvolumens oberhalb des Filtermittels) nimmt der resultierende Druck bei konstanter Rotordrehgeschwindigkeit kontinuierlich ab. Abbildung 3 zeigt den schematischen Aufbau des Filtrationsmoduls im Zentrifugalfeld. Mit fortlaufender Filtrationszeit nimmt der Abstand vom Rotationszentrum zur Oberfläche

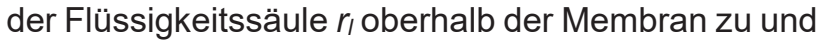
die eigentliche Höhe $\left(r_{m}-r_{l}\right)$ ab. Gemäß Gl. 1 nimmt somit der Druck bei konstanter Winkelgeschwindigkeit kontinuierlich ab.

$$
p(r)=\int_{r_{l}}^{r_{m}} \rho_{l} \omega^{2} r d r=\frac{\rho_{l} \omega^{2}}{2}\left(r_{m}^{2}-r_{l}^{2}\right)
$$

Zur Bestimmung des Filtermittelleerwiderstandes wird das FM mit einer reinen Flüssigkeit, vorzugsweise mit der kontinuierlichen Phase der Suspension, durchströmt. Der Druck $p$ ergibt sich aus dem radialen Abstand der Flüssigkeitssäule zum Rotationszentrum $r_{l}$, der Winkelgeschwindigkeit $\omega$, der Dichte des Fluides $\rho_{l}$ und dem radialen Abstand zum FM $r_{m}$. Zur Verwendung kommen sehr dünne FM, sodass deren Dicke vernachlässigt werden kann. Gl. 1 gilt nur bei Verwendung von reinen Flüssigkeiten. Die Filtration von Suspensionen führt zu einem Haufwerk aus abgelagerten Partikeln, dessen Gerüstdruck ebenfalls zu dem Druck aus GI. 1 additiv hinzukommt, 
bzw. die Geometrie des wirkenden Drucks beeinflusst.

Bei Filtration von Suspensionen werden dispergierte Partikel, welche meist größer sind als die Poren des FM auf dessen Oberfläche zurückgehalten. Die so entstandene Schicht wird als Filterkuchen (FK) bezeichnet. Der FK selbst baut sich mit fortschreitender Filtration zunehmend auf, sodass die kontinuierliche Phase einen stetig größer werdenden Widerstand überwinden muss.

Die Durchströmung poröser Medien wird durch die Darcy Gleichung beschrieben. Sie definiert den Volumenstrom $d V / d t$ als Quotient der gesamten Druckdifferenz $\Delta p$ und des gesamten Filterwiderstandes $R$ normiert mit der Viskosität $\eta$ der kontinuierlichen Phase der Dispersion (vgl. Gl. 2).

$$
\frac{d V}{d t}=\frac{\Delta p}{\eta R}
$$

Unter der Annahme eines inkompressiblen FK, ist der spezifische Filterkuchenwiderstand $\mathrm{r}_{\mathrm{FK}}$ eine lineare Funktion der Filterkuchenhöhe $h_{F K}$ und der Permeabilität $K_{F K}$ und die Durchströmung kann durch $\mathrm{Gl}$. 3 beschrieben werden:

$$
\frac{d V_{F K}}{d t}=\frac{K_{F K} \Delta p_{F K}}{\eta h_{F K}}
$$

Eine direkte Bestimmung von $\mathrm{r}_{\mathrm{FK}}$ ist durch PZF, wie in konventionellen Filternutschenversuchen, nicht möglich. Alternativ wird auf dem Gesamtwiderstand $R$ zurückgegriffen und lässt sich mit Gleichung 4 beschreiben. Der Filtermittelleerwiderstand und der spezifische Kuchenwiderstand sind additiv.

$$
\frac{d V}{d t}=\frac{\Delta p_{g e s}(t)}{\eta\left(r_{F K} h_{F K}(t)+R_{F M}\right)}
$$

Mithilfe der Gleichungen (2 - 4) lässt sich rFK bestimmen. Dafür wird zunächst der Filtermittelleerwiderstand mit der reinen Flüssigkeit bestimmt. In einem

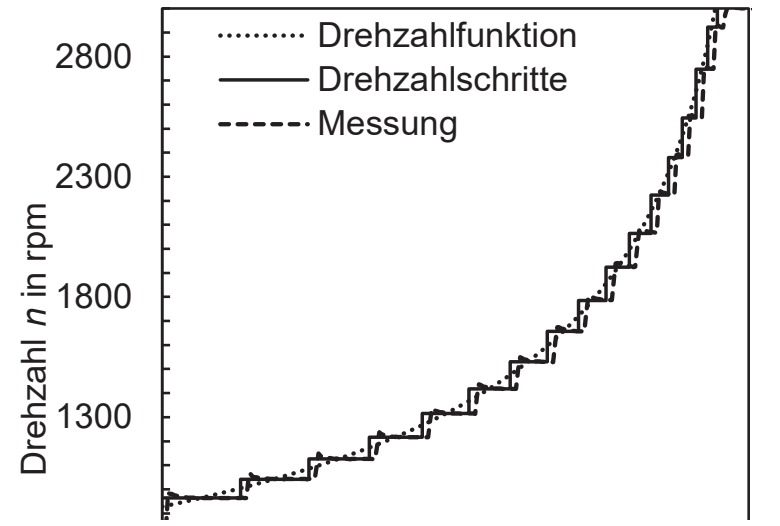

Abb. 4: Exemplarische Darstellung einer Drehzahlrampe für den Betrieb der PZF mit konstantem Druck zweiten Experiment wird die Suspension filtriert. Im letzten Experiment wird dann erneut mit der kontinuierlichen Phase filtriert, wo hierbei sowohl der Filtermittelwiderstand als auch der Filterkuchenwiderstand überwunden werden muss (siehe Abschnitt Ergebnisse).

Für Vergleichsmessungen wird eine konventionelle Filternutsche, für Fluidvolumina von etwa 250 Milliliter, verwendet. Die Filtermittelauflagefläche beträgt 20 Quadratzentimeter. Das Filtratvolumen wird gravimetrisch bestimmt.

Als FM dienen Polymer-Flachmembranen (Polypropylen) mit einem nominellen Porendurchmesser von 100 Nanometer. Aufgrund der Hydrophobizität werden diese vor den Versuchen mit Ethanol benetzt. Aufgrund der sehr dünnen (etwa 90 Mikrometer Dicke) FM wird das Filter zusätzlich von einem Supportmaterial (Maschenweite 105 Mikrometer) gestützt.

Als kontinuierliche Phase wird vollentsalztes Wasser verwendet. Die disperse Phase sind Vollglaskugeln mit einem mittleren Durchmesser von etwa 11 Mikrometer.

Um eine Vergleichbarkeit mit konventioneller Nutschenfiltration nach VDI 2762 zu erreichen, wird die
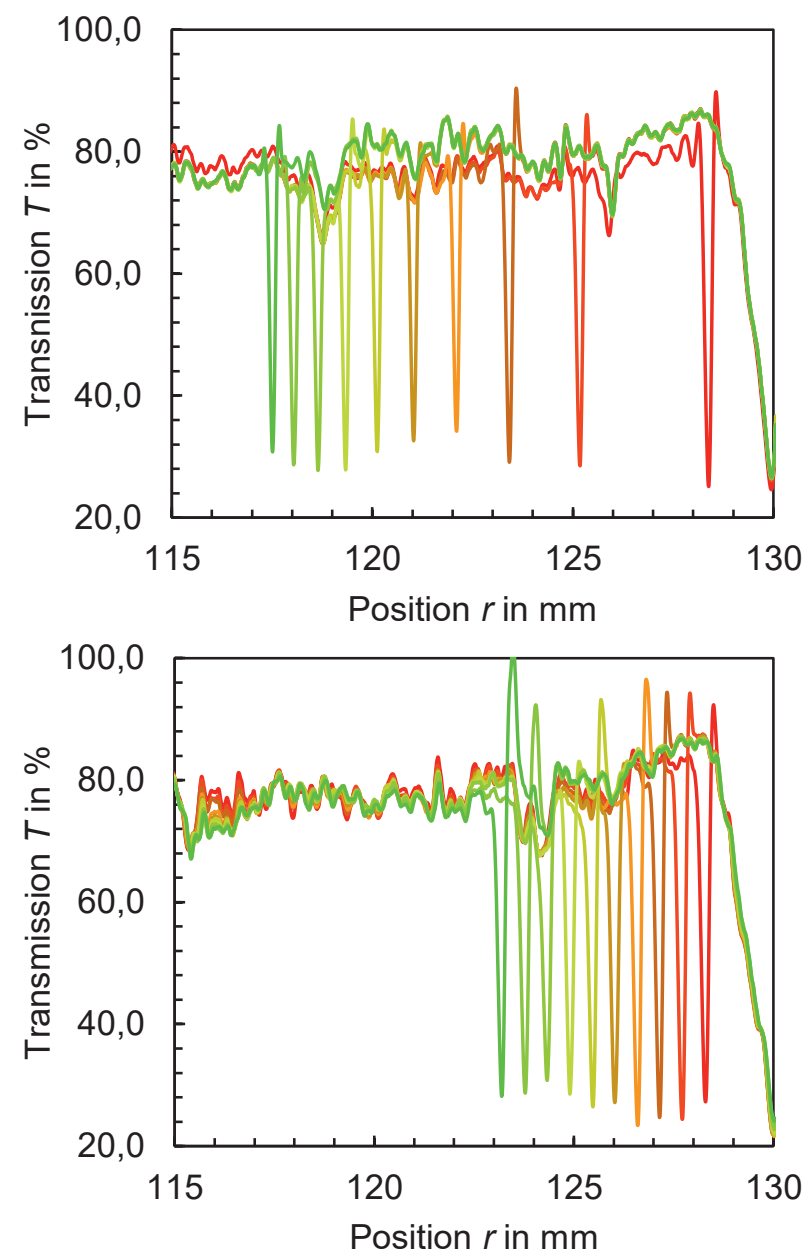

Abb. 5: Filtrationsfingerprints der PZF; zeitliche Profilentwicklung von Rot nach Grün; oben: konstante Rotordrehzahl; unten: konstanten Druck 


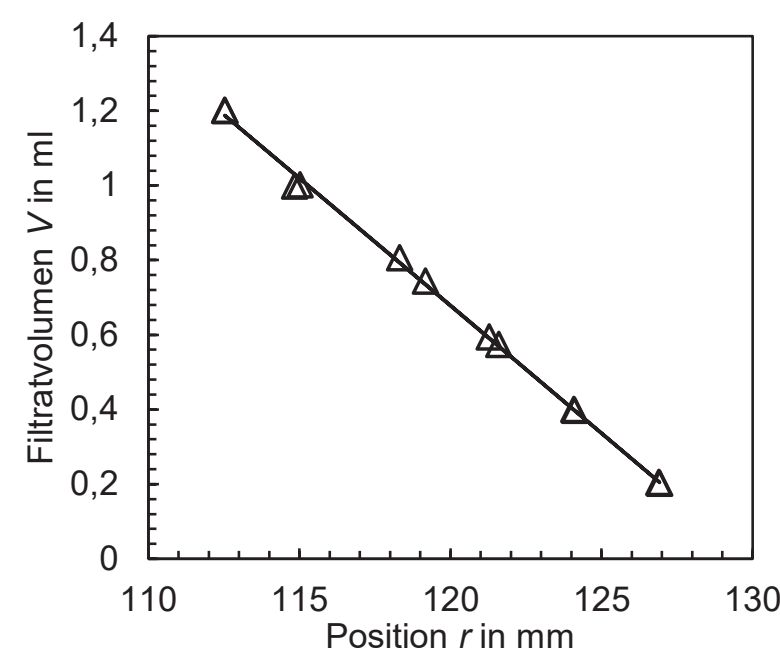

Abb. 4: Funktion zur Bestimmung des Filtratvolumens aus der Position des Meniskus

PZF mit einer kontinuierlich steigender Drehzahlrampe programmiert, um einen konstanten Überdruck einzustellen (Abb.4).

Abbildung 5 zeigt den Vergleich der Transmissionskurven bei Verwendung einer konstanten Drehzahl (oben) und eine stetig nachregelnde Drehzahl (unten). Die zeitliche Transmissionsprofilentwicklung ist von Rot (erstens Profil, Beginn der Zentrifugation) nach Grün (letztes Profil, Ende der Filtration). Die zeitlichen Abstände zwischen den Profilen sind identisch. Die örtlichen Abstände der lokalen Transmissionsminima jedes Profils in Abb. 5 oben nehmen mit zunehmender Filtrationszeit ab, gleichbedeutend mit einem Fluss und Druckabnahme. In der unteren Abbildung wurde der Druck konstant gehalten, die Abstände der Minima sind nahezu identisch, was auf einen über der Zeit konstanten Filtratstrom schließen lässt.

Zur Bestimmung des Filtratvolumens aus den Meniskuspositionen wurde eine Funktion erstellt, welche den Zusammenhang zwischen absoluter (Rotor)Radiusposition des Meniskus und der entsprechenden Filtratmenge herstellt (Abb. 6).

Abbildung 7 zeigt das Filtratvolumen über der Filtrationszeit für eine konstante Drehzahl (oben) und einer in-situ korrigierten Drehzahl (unten). Während bei der Verwendung von konstanter Rotordrehzahl (Abb. 7 oben) der Filtratfluss $d V / d t$ stetig über der Zeit (vgl. GI. 1) abnimmt, wird durch in-situ Drehzahlanpassung (Abb. 7 unten) ein nahezu konstantes $d V / d t$ erreicht und somit ein zeitlich quasi-konstanten Druckverlauf. Zum zeitlichen Ende des Experimentes fält der Druck ab, da die Drehzahl nicht weiter erhöht und konstant gehalten wird.
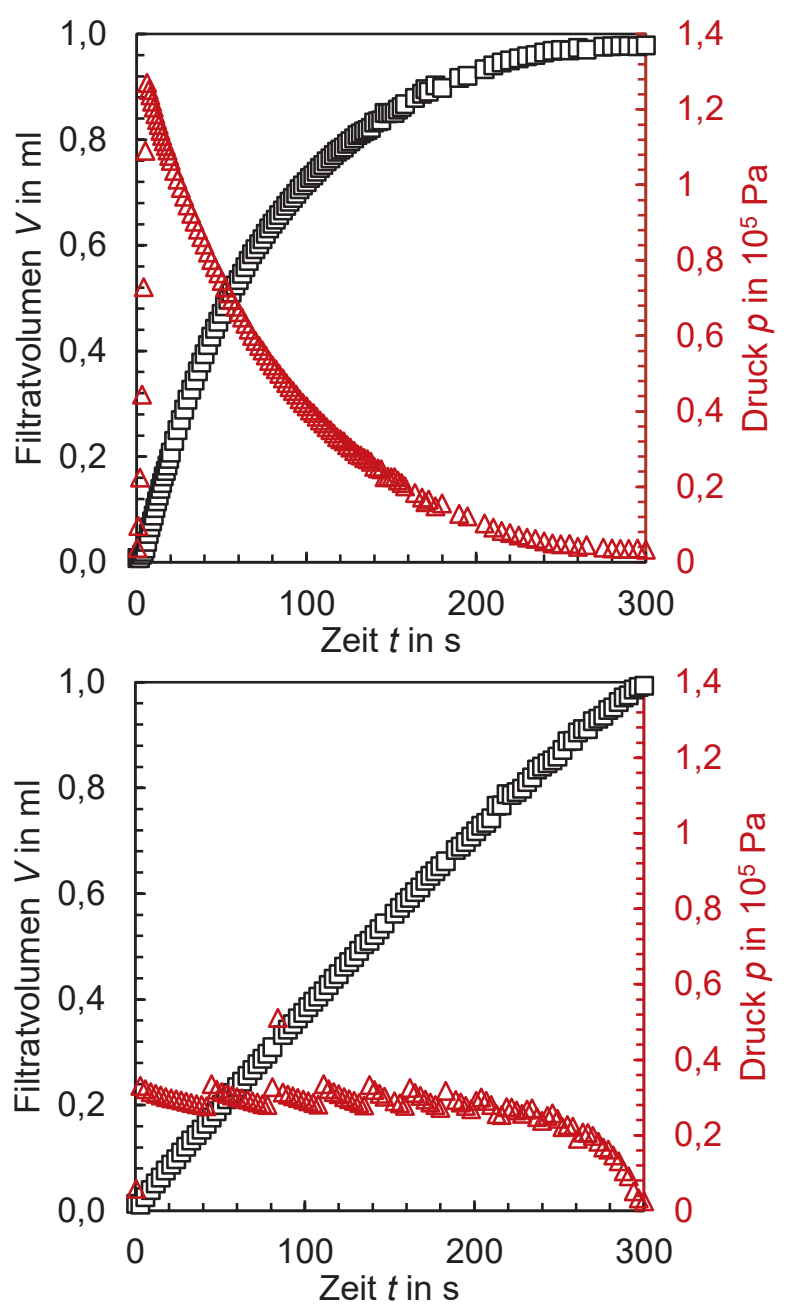

Abb. 5: Filtratvolumen (schwarz) und Druckverlauf (rot) über der Filtrationszeit in der PZF; oben: Betrieb mit konstanter Drehzahl; unten: Betrieb mit Drehzahlrampe

\section{Ergebnisse}

Zunächst wurde in der PZF der Filtermittelwiderstand bestimmt. Für die Versuche zur Bestimmung des Filtermittelwiderstandes mit konstanter Rotordrehzahl wurden Maximaldrücke von 2 bar realisiert. Für die Experimente mit konstantem Druck wurde ein durchschnittlicher Druck von 1,2 bar realisiert.

Die Ergebnisse durch Verwendung von konstantem Druck, sowie konstanter Drehzahl werden mit den Resultaten der Filternutsche verglichen. Der Vergleich zwischen den mit der Filternutsche und der PZF ermittelten Werten zeigt eine gute Übereinstimmung (Abb. 8). Je größer der quasikonstante Filtrationsdruck, desto größer werden die Streuungen der Messwerte in einer Messreihe. 


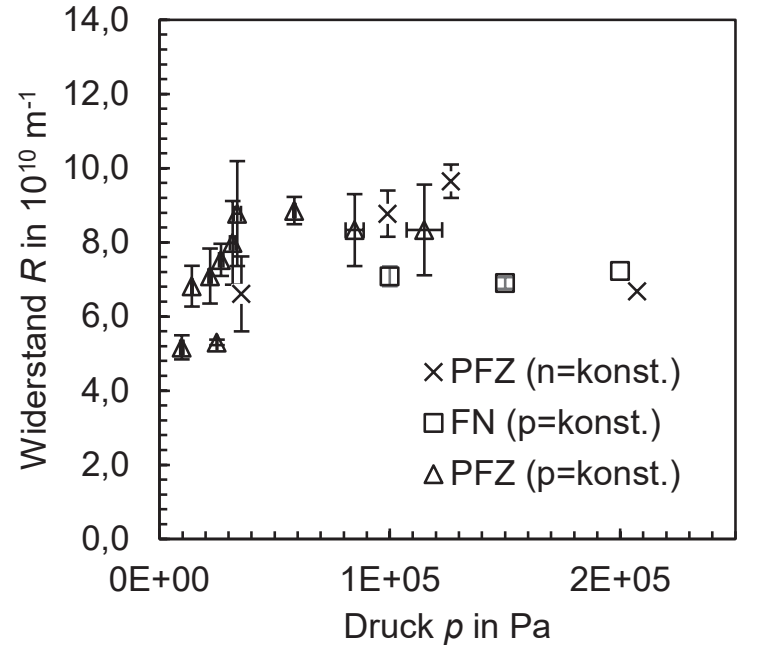

Abb. 6: Vergleich der Filtermittelwiderstände der verwendeten Methoden

Zur Bestimmung des Filtermittel- und Kuchenwiderstandes wurde das Experiment in drei aufeinander folgende Filtrationsteilprozesse in der PZF unterteilt. Der erste Teil des Experiments beinhaltet die Durchströmung des FM mit der kontinuierlichen Phase (Reinstwasser) zur Bestimmung des Filtermittelwiderstandes gemäß GI. 2. Im zweiten Schritt wurde die Filtration mit der Suspension durchgeführt. Dabei bildet sich der FK sukzessive auf und lässt den Gesamtwiderstand (vgl. Gl. 4) stetig steigen, bis sich alle Partikeln abgelagert haben und der FK voll ausgebildet ist. Der Durchströmungswiderstand erreicht einen konstanten Wert. In einem dritten Schritt wird erneut

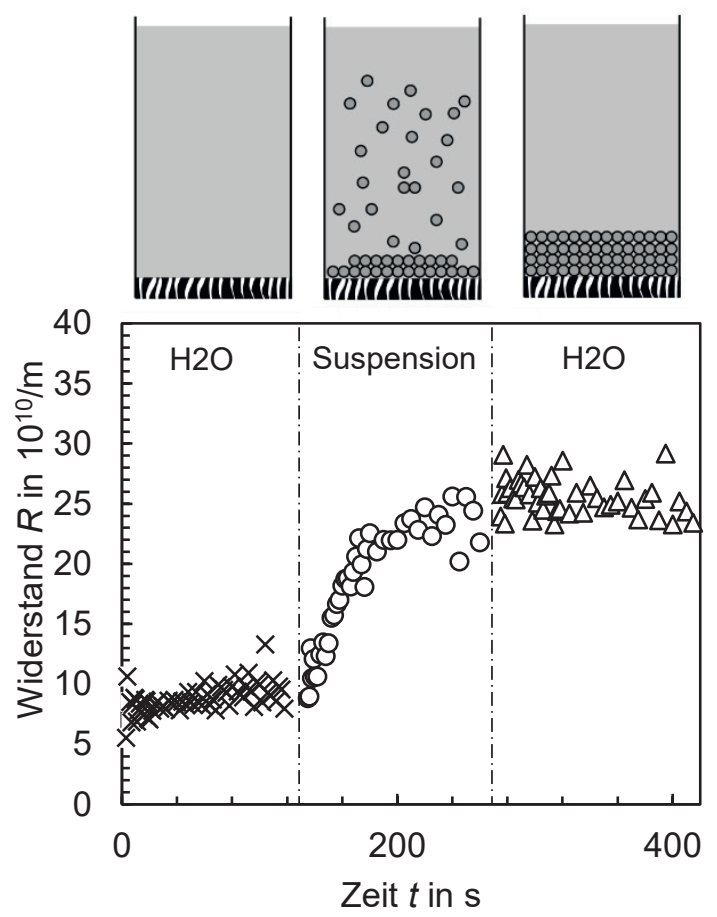

Abb. 9: Versuchsreihe zur Bestimmung des Filtermittel- und Kuchenwiderstandes die kontinuierliche Phase durch das FM und den zuvor gebildeten (nun statischen) FK gefiltert. Daraus kann dann der spez. Filterkuchenwiderstand gemäß $\mathrm{Gl} .3$ bestimmt werden.

Abbildung 9 zeigt den Gesamtwiderstand in Abhängigkeit der Zeit bei den drei aufeinander folgenden Prozessschritten für ein Experiment mit Glaskugeln.

Bei der Durchströmung mit reinem Fluid im ersten Schritt zeigt sich der erwartete zeitlich konstante Verlauf des Widerstands. Hier wird nur der Durchströmungswiderstand des FM erfasst. Nach dem Befüllen mit Suspension steigt der Gesamtwiderstand mit der Zeit linear an, was die Bildung eines inkompressiblen FK charakterisiert. In der zweiten Hälfte der Durchströmung mit der Suspension ist der FK schon fast vollständig ausgebildet und eine reine Durchströmung des gebildeten Haufwerks erzeugt einen konstanten Durchströmungswiderstand. Dies wird bei der Betrachtung des dritten Prozessschritts bestätigt, hier ergibt sich der gleiche konstante Durchströmungswiderstand.

Trotz der Entnahme der Küvetten und erneutes Befüllen mit Fluid/Suspension zeigt sich im Übergang zwischen den Prozessschritten eine sehr gute Übereinstimmung des reinen Filtermittel- bzw. kuchenwiderstands.

Abbildung 10 zeigt die nach der vorgestellten Methode ermittelten Filterkuchenwiderstände im Vergleich mit denen der Filternutsche. Hierbei wurde bei der Filternutsche ebenfalls eine Durchströmung des Filterkuchens mit reinem Fluid im Anschluss an die Kuchenfiltration durchgeführt.

Die Ergebnisse liegen alle in der gleichen Potenz, die bei den mit der Filternutsche ermittelten Widerstände aufgetretenen Messschwankungen deuten auf die Schwierigkeit der Bestimmung hin. Während die bei konstanter Drehzahl ermittelten Werte eher dazu neigen, den FK zu unterschätzen, können die bei konstantem Filtrationsdruck erzielten Ergebnisse bessere Übereinstimmungen liefern.

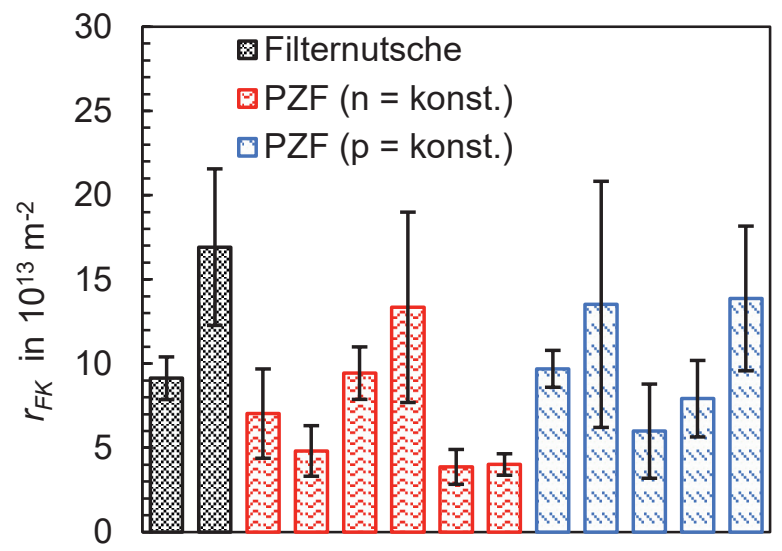

Abb. 10: Spezifischer Filterkuchenwiderstand der Glaskugel-Suspension bestimmt mit den verwendeten Methoden 


\section{Zusammenfassung}

Es wird ein neues miniaturisiertes Mehrproben-Photozentrifugalfiltrationsverfahren zur Bestimmung von Filtermittel- und Membranwiderständen mit und ohne Filterkuchenbildung beschrieben.

Die im Rahmen dieser Untersuchungen vorgestellten Ergebnisse mit konventioneller Nutschenfiltration und der analytischen Zentrifugalfiltration zeigen gute Übereinstimmungen. Die PZF liefert Ergebnisse in den gleichen Größenordnungen. Wird der Filtermittelwiderstand bei konstanter Drehzahl, also abnehmendem Filtrationsdruck bestimmt, ergeben sich gute Übereinstimmungen mit den aus den Filternutschenexperimenten erhaltenen Werten. Bei Kenntnis des Filtermittelwiderstands kann eine Drehzahlrampe erstellt werden, welche einen konstanten Filtrationsdruck erzeugt. Somit können die ermittelten Gesamtwiderstände mit den Ergebnissen der Filternutsche gut verglichen werden. Mithilfe einer erneuten Durchströmung des Filterkuchens mit der kontinuierlichen Phase konnte gezeigt werden, dass selbst bei den geringen Suspensionsmengen der Filterkuchenaufbau während der Versuchszeit vollständig abläuft.

Die PZF ist eine geeignete Methode zur schnellen, ökologischen und -nomischen Quantifizierung der Filtermedien bzw. -membranen sowie der Filtrierbarkeit von Dispersionen. Gerade für die Zielapplikationen (wertige Materialien, Gefahrstoffe) bietet dies deutliche Vorteile zur effizienten Entwicklung von neuen Materialien oder Prozessoptimierungen.

\section{Literatur}

[1] S. Ripperger, in Handbuch der Mechanischen Verfahrenstechnik (Ed: H. Schubert), John Wiley \& Sons, Hoboken 2003.

[2] Verein Deutscher Ingenieure, Mechanische FestFlüssig-Trennung durch Kuchenfiltration: Übersicht, Beuth Verlag GmbH, Berlin 71.04.40, 2006.

[3] Verein Deutscher Ingenieure, Filtrierbarkeit von Suspensionen: Bestimmung des Filterkuchenwiderstands, Vol.71.040.40 (2762 Blatt 2), Beuth Verlag $\mathrm{GmbH}$, Berlin 2010.

[4] D. Lerche, Comprehensive characterization of nano- and microparticles by in-situ visualization of particle movement using advanced sedimentation techniques, KONA 2019, 36, 156-186. DOI: 10.14356/kona.2019012.

[5] S. Boldt, D. Lerche, M. Loginov, Analytical PhotoCentrifugal Filtration (ACF): Membrane Resistance and Filterability, F\&S International Edition 2019, 15-21.

\section{Danksagung}

Die Autoren danken dem Bundesministerium für Wirtschaft und Technologie (KK5048402CM0 - PräLab - Entwicklung eines Verfahrens zur Prädiktion und Auslegung von industriellen Filtrationsprozessen basierend auf experimentellen Laboruntersuchungen mit analytischen multi-sample Photofiltrationszentrifugen) für die finanzielle Unterstützung. 